\title{
Contemporary ten-year outcomes for aorto-bi- femoral bypass in the endovascular era
}

\begin{abstract}
Background: The recently described "Covered Endovascular Reconstruction of the Aortic Bifurcation (CERAB)" technique challenges the role of open surgical bypass in the treatment of aorto-iliac occlusive disease. Complications and mortality after aortobi-femoral bypass have remained stable over the last three decades despite declining caseloads but there are few contemporary outcomes to enable direct comparison. We present 10 years of consecutive patients treated with aorto-bi-femoral bypass at a single tertiary centre
\end{abstract}

Methods: A retrospective review was performed of prospectively maintained operating theatre logbooks. Notes were reviewed to ascertain the indication for surgery in addition to demographic data. Cross-sectional pre-operative imaging was reviewed to determine the extent of the disease according to the TASC-II classification. Primary endpoints were graft patency and survival. Secondary endpoints were reinterventions, immediate and delayed complications. Results: Between 2004 and 2015, 185 patients (61\% male) with peripheral vascular disease (51\% Fontaine Class $2 \mathrm{~b}, 25 \%$ Class 3 , $21 \%$ Class 4 ) were treated. The median age was 62 years (range 37-82). 58\% were current smokers, $41 \%$ ex-smokers. $26 \%$ had undergone previous attempts at treatment (42/49 by endovascular means). Lesion morphology characterised by CT angiography included 1 TASC-II A, 9 TASC-II B, 25 TASC-II C and 97 TASC-II D lesions. Median follow up was 67 months. Thirty-day mortality was $4.3 \%$. There were 28 major complications (15\%), including 7 early limb occlusions requiring reintervention. Patency was $94 \%$ at 1 year, $88 \%$ at 3 years, $82 \%$ at 5 years and $79 \%$ at 10 years In $31 \%$ of cases the limbs of the graft were landed distal to the common femoral artery. To date there have been 9 cases of graft infection, 5 requiring explantation. Survival was $94 \%, 81 \%$ and $64 \%$ at 1,5 and 10 years respectively. Conclusions: Open surgery cannot compete with low 30-day morbidity and mortality rates reported with endovascular techniques. However, patency in our series exceeds much of the historical literature for this procedure despite the graft landing distal to the common femoral artery in one third of patients. Comparing our data to the available 1 and 2 -year CERAB patency rates, our rates exceed endovascular patency by over $5 \%$ at both 1 and 3 years. Secondary patency for CERAB can be obtained but longer-term follow-up for endovascular techniques have yet to be reported. The "Gold Standard" remains a subject for debate.

Keywords: aorto-iliac disease, aorto-bi-iliac/femoral bypass, endovascular, Morganella morganii, Enterococcus faecalis, Candida zeylanoides, Streptococcus anginosus, Candida tropicalis and Escherichia coli, graft infection, gold standard, intraoperative haemorrhage
Volume 6 Issue 6 - 2018

Frances Kent,' Yen Ming Chan,' Diane Hildebrand,' Russell Jamieson,' Bryce

Renwick'

'Department of Surgery, Aberdeen Royal Infirmary, Scotland

Correspondence: Bryce Renwick, Consultant Vascular and Endovascular Surgeon, Aberdeen Royal Infirmary, Scotland, UK AB25 2ZN, Email b.renvick@nhs.net

Received: October 24, 2018 | Published: November 16, 2018
Abbreviations: $\mathrm{ABG}$, arterial blood gas; CERAB, covered endovascular reconstruction of the aortic bifurcation; TASC, total administrative services corporation; SPSS, statistical package for the social sciences.

\section{Introduction}

The traditional approach to occlusive aorto-iliac disease is either an extra-anatomical axillo-femoral bypass or anatomical aorto-biiliac/femoral bypass (ABG). In this era of advancing endovascular techniques, the Covered Endovascular Reconstruction of the Aortic Bifurcation (CERAB) has been presented as a minimally invasive alternative to the surgical approach ${ }^{1}$. There have been a limited number of publications of recent outcomes for the surgical approach ${ }^{2}$, some simply meta-analyses of historical data. ${ }^{3,4}$ There is evidence that a decreasing number of open surgical bypasses are being performed for severe aorto-iliac disease. ${ }^{5}$ A recent national registry analysis demonstrated that over the 20-year period from 1993-2013 the number of bifurcated aortic grafts performed for chronic ischaemia reduced by two thirds. ${ }^{6}$ As a tertiary unit that still performs a relatively high number of these operations, we analysed a large cohort of ABG cases with up to a decade of follow-up. We propose that outcomes from newer endovascular techniques are compared to contemporaneous data such as this.

\section{Materials and methods}

This study complied with the Declaration of Helsinki. Patient consent and institutional review board approval was not required for this retrospective study. A retrospective review was performed of prospectively maintained operating theatre logbooks to capture all ABG operations performed between 2004 and 2015. Notes were reviewed to ascertain the indication for surgery in addition 
to demographic data. Cross-sectional pre-operative imaging was reviewed to determine the extent of the disease according to the TASC-II classification. ${ }^{7}$ Primary endpoints were graft patency and survival. Secondary endpoints were reinterventions, immediate and delayed complications. SPSS software was used to analyze patency and survival rates using Kaplan Meier survival analysis (Statistical Package for the Social Sciences, Inc., Chicago, IL).

\section{Results}

Between January 2004 and December 2015185 patients underwent an ABG. Median follow up was 67 months (range 10 - 149). Demographics are shown in Table 1. Pre-operative symptoms were scored according to the Fontaine classification ${ }^{8}$ : 51\% had Fontaine Class 2 b symptoms, $25 \%$ Class 3 and $21 \%$ Class 4 . Just over a quarter $(26 \%)$ had undergone previous attempts at revascularisation, 42/49 by endovascular means (10 iliac angioplasties, 32 iliac stents). The severity of disease according to symptoms and lesion morphology (assessed in patients undergoing an ABG as a primary intervention and scored according to TASC-II classification ${ }^{7}$ is shown in (Table 1) (Table 2).

Table I Patient demographics

\begin{tabular}{ll}
\hline Age & Median 61.8 years (range 36-82) \\
\hline Male & $60.50 \%$ \\
Diabetic & $13.50 \%$ \\
Current smoker & $57.80 \%$ \\
Ex smoker & $40.50 \%$ \\
Obese $(\mathrm{BMI}>30)$ & $16.20 \%$ \\
\hline
\end{tabular}

Table 2 Indication for treatment

\begin{tabular}{ll}
\hline & $\%$ of patients \\
\hline TASC-II A lesion & $0.80 \%$ \\
TASC-II B lesion & $6.80 \%$ \\
TASC-II C lesion & $18.90 \%$ \\
TASC-II D lesion & $73.50 \%$ \\
Fontaine Class 2b (severe claudication & $51 \%$ \\
$<200 y d s)$ & \\
Fontaine Class 3 (rest pain) & $25 \%$ \\
Fontaine Class 4 (tissue loss) & $21 \%$ \\
\hline
\end{tabular}

Twenty-six (14\%) of patients required concomitant surgical procedures, including 12 distal bypasses and 11 femoral endarterectomies. In 55 ( $31 \%$ ) of cases the aortic bypass graft had to be landed distal to the common femoral artery due to occlusive femoral disease. Thirtyday mortality was $4.3 \%$. Causes of death included intraoperative haemorrhage, ${ }^{2}$ multi-organ failure, ${ }^{1}$ ischaemic bowel, ${ }^{1}$ myocardial ischaemia ${ }^{2}$ and pneumonia. ${ }^{1}$ There were 6 late deaths related to the bypass graft, occurring $17-93$ months after surgery ${ }^{2}$ ( 2 due to graft sepsis, 4 due to limb ischaemia). There were 28 major complications (15\%), including 7 early limb occlusions requiring reintervention. All 30-day complications are shown in (Table 3).

Long term complications included 11 incisional herniae (5 requiring repair) and 9 cases of graft infection (5 grafts explanted). Of the explanted grafts, one had no growth of pathogens (despite the graft being exposed in the groin), the other explanted graft specimens grew pathogens including Morganella morganii, Enterococcus faecalis, Candida zeylanoides, Streptococcus anginosus, Candida tropicalis and Escherichia coli. The grafts were explanted a median of 19 months postoperatively (range 5-29 months). Of the five explanted grafts, three had been performed for critical limb ischaemia (two for short distance claudication). Kaplan Meier curves demonstrating patency are shown in Figure 1. Patency was $94 \%$ at 1 year, $88 \%$ at 3 years, $82 \%$ at 5 years and $79 \%$ at 10 years. Kaplan Meier curves demonstrating survival are shown in Figure 2. Survival was 94\%, $81 \%$ and $64 \%$ at 1,5 and 10 years respectively (Figure 1) (Figure 2).

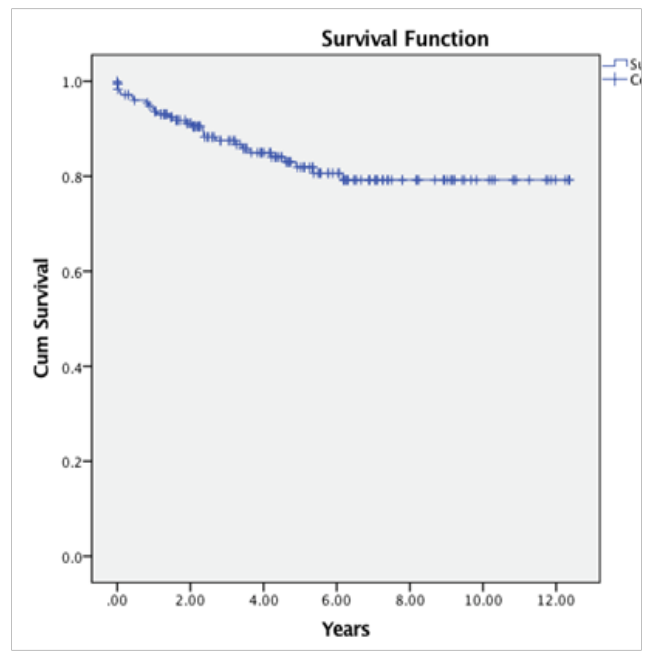

Figure I Primary patency



Figure 2 Survival

Table 3 Complications

\begin{tabular}{ll}
\hline Complication & Number of patients \\
\hline $\begin{array}{l}\text { Wound complication (infection, } \\
\text { seroma, haematoma) }\end{array}$ & 14 \\
$\begin{array}{l}\text { Urinary tract infection / urinary } \\
\text { retention }\end{array}$ & 4 \\
$\begin{array}{l}\text { Respiratory complication (infection, } \\
\text { embolism, oedema) }\end{array}$ & 44 \\
Early graft occlusion & 7
\end{tabular}


Table Continued

\begin{tabular}{ll}
\hline Complication & Number of patients \\
\hline $\begin{array}{l}\text { Cardiac complication (ischaemia, } \\
\text { atrial fibrillation) }\end{array}$ & 7 \\
\hline Gl complication (ileus, ischaemic & 24 \\
bowel) & 1 \\
Line sepsis & 5 \\
Acute kidney injury & 2 \\
Stroke & 2 \\
Acute delirium & \\
\hline
\end{tabular}

\section{Discussion}

Anatomical bypass has previously been the gold standard treatment for aortoiliac occlusive disease, with better patency rates than non-anatomical bypass. ${ }^{9}$ Given the substantial perioperative morbidity of the operation and significant co-morbidities of this group ${ }^{4}$ endovascular revascularisation has been proposed as an alternative. Initial reports of iliac stenting showed a large variation in outcome, with primary patency results from $70 \%$ - 97\% at one year and $60-86 \%$ at 5 years. ${ }^{4}$ Covered stents were introduced after initial interventions with bare metal stents, and the randomized COBEST trial demonstrated superiority of this approach. ${ }^{10}$ Finally, CERAB has been introduced as an attempt to overcome the anatomical and physiological disadvantages of kissing stents - for example the perfused 'dead lumen' space around the stents and mismatch of the overlap distance of the stents in the aorta. ${ }^{11,12}$ Initial results of the CERAB procedure are starting to be reported. ${ }^{1}$

Acknowledging the interest in, and potential role of, endovascular revascularisation, as a centre that regularly performs aorto-bi-femoral/ iliac grafts we analysed our patency and survival outcomes to provide contemporary long-term results for comparison with CERAB. We hypothesised that with improved medical therapy of atherosclerotic disease, optimised pre-operative risk factor management and modern anaesthetic and post-operative care the results may even be superior to previously reported results. A meta-analysis of published results from ABGs over three decades published in 1997 demonstrated a decrease in perioperative mortality and systemic morbidity over time ${ }^{13}$ although a more recent 20 -year review published in 2015 documented no significant improvement in 30-day mortality or major complication rates. ${ }^{6}$ Our perioperative mortality was comparable to previous reports. ${ }^{3,13-15}$ Our total 30 day morbidity rate was higher than previously reported at $47 \%$, but once minor complications were excluded the major morbidity rate $(16 \%)$ was within the range previously reported by a large meta-analysis ${ }^{13}$ and the more recent 20 year review from Denmark in which only "elective" patients with chronic ischaemia were included. Of note our graft infection rate was higher than reported in previous cohorts $(5.9 \%$ compared to $0.8 \%-1.6 \%))^{3,16}$ This may be partially attributable to the long follow up period - graft explantation was required up to 29 months after initial surgery. Alternatively, our series includes ABG grafts inserted in emergency cases in patients with reduced healing potential and concurrent co-morbidity. Two of the nine patients with graft infection were diabetic. Interestingly though the number of obese patients remained static throughout the period studied $(16 \%)$, the number of diabetic patients undergoing an ABG increased from $9 \%$ in the years 2004-2009 to $16 \%$ from 2009-2015 ( $\mathrm{p}=0.35$ ). This, combined with the decreasing number of ABG grafts performed for potentially more severe atherosclerotic disease, may contribute towards increasing post-operative complications, such as graft infection.
We demonstrated that our patency rates compared favourably to results reported of a recent cohort ${ }^{2}$ and to more historic data. ${ }^{13,15,16}$ This is despite potentially more challenging cases being operated on as endovascular techniques are offered for less extensive disease and surgical repair is reserved for more severe disease. ${ }^{6} \mathrm{~A}$ recent meta-analysis could support this hypothesis - results demonstrated a decline in 5-year patency rate from $88.4 \%$ in the 1970 s to $76.1 \%$ (p $=0.03$ ) for results after the millennium, ${ }^{3}$ which was not explained by differences in patient characteristics. Overall survival rates of $64 \%$ at ten years reflect the age and co-morbidities of this patient group. It has been shown by recent reports that overall survival rates have improved since historical reports, ${ }^{3,6}$ and our data is in keeping with this. Although only initial results have been reported for the CERAB technique, the primary patency rates are lower than those in our cohort ( $87.3 \%$ at 1 year, $82.3 \%$ at 3 years), though the secondary patency rates are higher $\left(95.0 \%\right.$ at 1 and 3 years). ${ }^{1}$ Of note, the indication for intervention (i.e. Fontaine classification) of the patients in our cohort was more severe than in the cohort undergoing a CERAB procedure.

In the cohort of initial patients undergoing CERAB, secondary interventions to restore patency were endovascular in all but four cases, where surgical thrombectomy ${ }^{2}$ or bypass ${ }^{2}$ was required. Two of these patients had impaired outflow contributing to stent occlusion. The authors concluded that any distal stenosis that might impair outflow of the stents should be treated aggressively. ${ }^{1}$ Previous analysis of kissing stents and iliac stenting also pointed towards the influence of distal outflow on the patency of stents, ${ }^{4}$ and simultaneous infrainguinal bypass has also been associated with improved patency of surgical aortoiliac bypasses. ${ }^{14}$ In our cohort $12 \%$ of patients required a concomitant outflow procedure, which would have to be considered in planning revascularisation of the aorto-iliac segment. Perhaps even more significant however is the one third of patients in our study where the ABG graft limbs were landed distal to the common femoral artery. Such patients typically had advanced peripheral vascular disease with femoral occlusion and/or superficial femoral occlusion. In these cases, the profunda femoral artery was the preferred landing vessel with the aim of surgery to relieve rest pain by profunda revascularisation. For these patients to undergo any endovascular solution a common femoral reconstruction may well have been required to facilitate access and then the patency of a stented iliac system running in to profunda alone is much more debatable. The complication rates of open treatment of aorto-iliac disease are a main driver of the search for an endovascular solution. Our data, though comparable or favourable to historic data, demonstrates higher mortality and major complication rate compared to the CERAB group. There were a greater number of procedural related complications in the endovascular group (14.6\%), such as vessel dissection or rupture, though these were also managed endovascularly. ${ }^{1}$

\section{Conclusion}

Our data demonstrates a persistently significant level of postoperative complications and mortality from ABG surgery, though long-term patency is better than endovascular strategies. This longterm patency was despite the limbs of the ABG graft landing distal to the common femoral artery in one third of patients. The high number (14\%) of concomitant outflow procedures performed at ABG surgery highlights the extent and severity of this group of patients' atherosclerotic disease and may contribute to poor stent patency in endovascular cohorts. The need for surveillance, secondary interventions and perhaps most crucially good run-off are currently 
disadvantages of the endovascular approach. When deciding between open and endovascular revascularisation, infrainguinal disease should be assessed and addressed aggressively. In patients with multilevel disease open surgical revascularisation may still be the "gold standard".

\section{Acknowledgement}

Kent F: Concept, data collection, data analysis, writing, Chan Y: Data collection, review, Hildebrand D: Data analysis, writing, review, Jamieson R: Concept, writing, review.

\section{Conflicts of Interest}

Author declares that there is no conflict of interest.

\section{References}

1. Grimme F, Goverde P, Verbruggen P, et al. First Results of the Covered Endovascular Reconstruction of the Aortic Bifurcation (CERAB) Technique for Aortoiliac Occlusive Disease. J Vasc Surg. 2015;62(5):1371-1372.

2. Lee G, Yang S, Park K, et al. Ten-year outcomes after bypass surgery in aortoiliac occlusive disease. J Korean Surg Soc. 2012;82(6):365-369.

3. Chiu K, Davies R, Nightingale P, et al. Review of direct anatomical open surgical management of atherosclerotic aorto-iliac occlusive disease. Eur J Vasc Endovasc Surg. 2010;39(4):460-471.

4. Jongkind V, Akkersdijk GJ, Yeung KK, et al. A systematic review of endovascular treatment of extensive aortoiliac occlusive disease. $J$ Vasc Surg. 2010;52(5):1376-1383.

5. Kashyap VS, Pavkov ML, Bena JF, et al. The management of severe aortoiliac occlusive disease: endovascular therapy rivals open reconstruction. J Vasc Surg. 2008;48(6):1451-1457.

6. Bredahl K, Jensen LP, Schroeder TV, et al. Mortality and complications after aortic bifurcated bypass procedures for chronic aortoiliac occlusive disease. J Vasc Surg. 2015;62(1):75-82.

7. Norgren L, Hiatt WR, Dormandy JA, et al. Inter-society consensus for the management of peripheral arterial disease (TASC II). Eur J Vasc Endovasc Surg. 2007;33(1):S1-S75.

8. Fontaine R, Kim M, Kieny R. Die chirurgische Behandlung der peripheren Durchblutungsstörungen. Helv Chir Acta. 1954;21(56):499-533.

9. Skelly CL, Milner R. Difficult Decisions in Vascular Surgery. Springer; 2017.

10. Mwipatayi BP, Thomas S, Wong J, et al. A comparison of covered vs bare expandable stents for the treatment of aortoiliac occlusive disease. $J$ Vasc Surg. 2011;54(6):1561-1570.

11. Greiner A, Muhlthaler H, Neuhauser B, et al. Does stent overlap influence the patency rate of aortoiliac kissing stents? J Endovasc Ther. 2005;12(6):696-703.

12. Sharafuddin MJ, Hoballah JJ, Kresowik TF, et al. Long-term outcome following stent reconstruction of the aortic bifurcation and the role of geometric determinants. Ann Vasc Surg. 2008;22(3):346-357.

13. de Vries SO, Hunink MG. Results of aortic bifurcation grafts for aortoiliac occlusive disease: a meta-analysis. J Vasc Surg. 1997;26(4):558-569.

14. Davidovic L, Vasic D, Maksimovic R, et al. Aortobifemoral grafting: factors influencing long-term results. Vascular. 2004;12(3):171-178.

15. Poulias GE, Polemis L, Skoutas B, et al. Bilateral aorto-femoral bypass in the presence of aorto-iliac occlusive disease and factors determining results. Experience and long term follow up with 500 consecutive cases. J Cardiovasc Surg (Torino). 1985;26(6):527-538.

16. Szilagyi DE, Elliott JP, Smith RF, et al. A thirty-year survey of the reconstructive surgical treatment of aortoiliac occlusive disease. $J$ Vasc Surg. 1986;3(3):421-436. 